\title{
Disparities in SARS-CoV-2 positivity among pregnant patients with limited English proficiency
}

(c) The Author(s), under exclusive licence to Springer Nature America, Inc. 2021

Journal of Perinatology (2021) 41:2564-2565; https://doi.org/10.1038/s41372-021-01148-w

\section{TO THE EDITOR}

Racial/ethnic disparities in severe acute respiratory syndrome coronavirus 2 (SARS-CoV-2) infection are well documented across the United States [1], including in pregnancy [2]. Limited English proficiency (LEP) widens health disparities [1], but less is known about its association with pregnancy outcomes. Given that SARSCoV-2 in pregnancy can cause severe disease [2], we examined the relationship between LEP and SARS-CoV-2 positivity in pregnancy.

We calculated rates of SARS-CoV-2 positivity among pregnant patients in GeoBirth, an ongoing cohort of all births at two Penn Medicine hospitals in Philadelphia, PA [3]. The electronic health record indicator "need for language interpreter," was used as a proxy measure for LEP. The study period was from the onset of universal SARS-CoV-2 screening at delivery with real-time reverse transcriptase-polymerase chain reaction testing at each hospital (1 April 2020 and 13 April 2020) through 31 January 2021. Deliveries $\geq 20$ weeks' gestation were included. Any positive test up until the time of delivery was counted, including positive tests conducted at community sites. We compared positivity rates by LEP status overall and stratified by Latinx ethnicity using two-sided chi-square tests and multivariable-adjusted log binomial regression to calculate risk ratios. Covariates that confounded the association of LEP with SARS-CoV-2 positivity by $>10 \%$ were retained. This study was approved by the University of Pennsylvania Institutional Review Board.

Among the 7298 eligible patients, $349(4.8 \%)$ had a positive SARS-CoV-2 test by delivery (Table 1 ) and 291 patients had LEP (23.4\% of Latinx patients and $2.1 \%$ of non-Latinx patients). Among patients with LEP, $12.4 \%$ tested positive compared to $4.5 \%$ of patients without LEP $(P<0.0001)$. The association between LEP and SARS-CoV-2 persisted after adjustment for race/ethnicity and insurance (adjusted relative risk (aRR) 1.99, 95\% confidence interval (Cl): 1.35-2.85; Fig. 1). Latinx patients were more likely to have a positive test than non-Latinx patients ( 9.8 vs. $4.3 \%, P<0.0001$ ). Within Latinx patients, those with LEP were more likely to test positive than those without LEP (19.6 vs. $6.8 \%, P<0.0001$, aRR $2.49,95 \% \mathrm{Cl}: 1.54-4.05)$. Among non-Latinx patients, LEP was not associated with SARS-CoV-2 positivity. The interaction between Latinx ethnicity and LEP was significant $(P=0.02)$.

Patients with LEP had an increased risk of testing positive for SARS-CoV-2 during pregnancy in two Philadelphia hospitals. In stratified analyses, the association was only positive among Latinx patients. LEP creates barriers to receipt and understanding of individual healthcare as well as public health information [1]. Latinx populations may be at particular risk of SARS-CoV-2 because of overrepresentation in essential jobs requiring face-to-face interactions, reliance on public transportation, and multi-generational living [4]. Healthcare access
Table 1. Demographic differences for pregnant patients with and without a positive SARS-CoV-2 test.

\begin{tabular}{|c|c|c|c|}
\hline Characteristics & $\begin{array}{l}\text { SARS-CoV-2 } \\
\text { positive } \\
n \text { (row percents }\end{array}$ & $\begin{array}{l}\text { No documented } \\
\text { positive test }\end{array}$ & $P$ value \\
\hline All participants & $349(4.8)$ & $6949(95.2)$ & \\
\hline Age (years) & & & $<0.0001$ \\
\hline$<25$ & $89(7.0)$ & $1178(93.0)$ & \\
\hline $25-<35$ & $202(4.8)$ & 4048 (95.2) & \\
\hline$\geq 35$ & $58(3.3)$ & $1723(96.7)$ & \\
\hline Race & & & $<0.0001$ \\
\hline Black & $224(6.7)$ & $3120(93.3)$ & \\
\hline White & $99(3.2)$ & 3006 (96.8) & \\
\hline Asian/other/unknown & $26(3.1)$ & $823(96.9)$ & \\
\hline Ethnicity ${ }^{\mathrm{a}}$ & & & $<0.0001$ \\
\hline Latinx & $64(9.8)$ & $591(90.2)$ & \\
\hline Non-Latinx & $284(4.3)$ & $6337(95.7)$ & \\
\hline Insurance & & & $<0.0001$ \\
\hline Private & $124(3.0)$ & $3990(97.0)$ & \\
\hline Public & $225(7.1)$ & $2959(92.9)$ & \\
\hline Pre-pregnancy BMI & & & $<0.0001$ \\
\hline$<25$ & $114(3.5)$ & 3119 (96.5) & \\
\hline $25-<30$ & $83(4.7)$ & $1674(95.3)$ & \\
\hline$\geq 30$ & $130(6.6)$ & $1829(93.4)$ & \\
\hline Marital status & & & $<0.0001$ \\
\hline Married & $103(2.8)$ & $3559(97.2)$ & \\
\hline Not married & $246(6.8)$ & 3390 (93.2) & \\
\hline Smoked during pregnancy ${ }^{c}$ & & & 0.50 \\
\hline Yes & $13(3.8)$ & $326(96.2)$ & \\
\hline No & $293(4.8)$ & $5820(95.2)$ & \\
\hline Parity & & & 0.0001 \\
\hline 0 & $117(3.6)$ & 3126 (96.4) & \\
\hline$>0$ & $232(5.7)$ & $3823(94.3)$ & \\
\hline $\begin{array}{l}\text { Limited English } \\
\text { proficiency }\end{array}$ & $36(12.4)$ & $255(87.6)$ & \\
\hline Latinx & $30(19.6)$ & $123(80.4)$ & 0.0004 \\
\hline Non-Latinx & $6(4.4)$ & 131 (95.6) & \\
\hline $\begin{array}{l}\text { No limited English } \\
\text { proficiency }\end{array}$ & $313(4.5)$ & 6694 (95.5) & \\
\hline Latinx & $34(6.8)$ & 468 (93.2) & 0.03 \\
\hline Non-Latinx & $278(4.3)$ & $6206(95.7)$ & \\
\hline \multicolumn{4}{|c|}{$\begin{array}{l}\text { a } 22 \text { patients declined to provide an ethnicity. } \\
\text { b } 349 \text { patients missing BMI. } \\
\text { c } 846 \text { patients missing smoking. } \\
\text { dOverall chi-square test comparing proportions of patients with and } \\
\text { without limited English proficiency had a } P \text { value of }<0.0001 \text {. }\end{array}$} \\
\hline
\end{tabular}

among Latinx LEP communities can be additionally limited by socio-political barriers [4]; Latinx patients with LEP are more likely to be underinsured than non-Spanish-speaking patients with LEP [5]. Although data on outcome variation by LEP status 


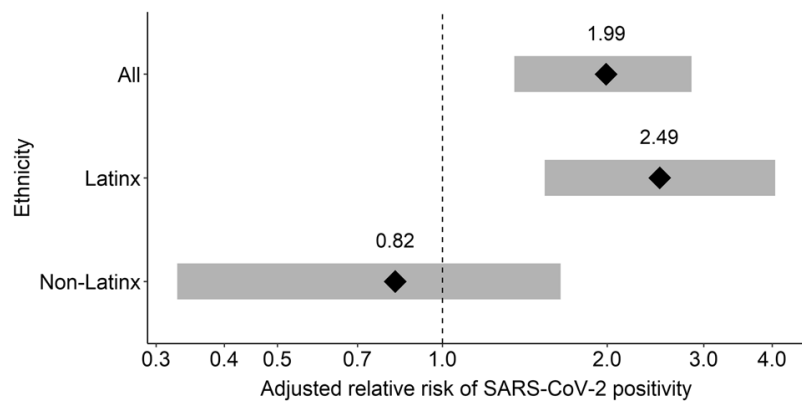

Fig. 1 Adjusted relative risk of SARS-CoV-2 positivity in patients with limited English proficiency as compared to patients without limited English proficiency. The interaction between Latinx ethnicity and limited English proficiency was significant $(P=0.02)$. Models were adjusted for race/ethnicity and insurance status. $X$ axis is on the logarithmic scale.

is scarce, there is some evidence that Latinx patients with LEP may be at higher risk for poor health than patients with LEP of different racial/ethnic backgrounds [6]. Our findings indicate that, for Latinx patients, language barriers combine with ethnicity to create increased risk of positivity in a way that is different for non-Latinx patients.

Study limitations include low representation of other races/ ethnicities, small sample size among non-Latinx patients with LEP, use of a single health system, and reliance on a previously used proxy for LEP. We also only had information on positive (not negative) community-based tests and could not capture patients with undiagnosed SARS-CoV-2 early in pregnancy who then tested negative at delivery. However, these limitations would have likely biased our findings toward the null, as we may have undercounted patients with high risk of infection and increased barriers to testing.

Given the ongoing disproportionate impact of SARS-CoV-2 infection on Latinx communities [1], our data highlight the need to improve pandemic public health measures among Latinx populations with LEP overall and within pregnancy. This may include collaboration with trusted community-based organizations [4] to improve testing, contact tracing and vaccine efforts, and improved delivery of language-concordant information and healthcare [1].

Diana Montoya-Williams $\mathbb{D}^{1 凶}$, Anne M. Mullin ${ }^{2}$, Sara C. Handley ${ }^{1}$, Dustin D. Flannery ${ }^{1}$, Scott A. Lorch ${ }^{1}$, Michal A. Elovitz (iD) ${ }^{2}$ and Heather H. Burris ${ }^{1}$

${ }^{1}$ Division of Neonatology, The Children's Hospital of Philadelphia, Philadelphia, PA, USA. 'Maternal and Child Health Research Center, University of Pennsylvania Perelman School of Medicine, Philadelphia, PA, USA. ${ }^{凶}$ email: montoyawid@chop.edu

\section{REFERENCES}

1. Diamond LC, Jacobs EA, Karliner L. Providing equitable care to patients with limited dominant language proficiency amid the COVID-19 pandemic. Patient Educ Couns. 2020;103:1451-2.

2. Zambrano LD, Ellington S, Strid P, Galang RR, Oduyebo T, Tong VT, et al. Update: characteristics of symptomatic women of reproductive age with laboratoryconfirmed SARS-CoV-2 infection by pregnancy status - United States, January 22October 3, 2020. MMWR Morb Mortal Wkly Rep. 2020;69:1641-7.

3. Handley SC, Mullin AM, Elovitz MA, Gerson KD, Montoya-Williams D, Lorch SA, et al. Changes in preterm birth phenotypes and stillbirth at 2 philadelphia hospitals during the SARS-CoV-2 pandemic, March-June 2020. JAMA. 2021;325:87-9.

4. Clark E, Fredricks K, Woc-Colburn L, Bottazzi ME, Weatherhead J. Disproportionate impact of the COVID-19 pandemic on immigrant communities in the United States. PLoS Negl Trop Dis. 2020;14:e0008484.

5. Foiles Sifuentes AM, Robledo Cornejo M, Li NC, Castaneda-Avila MA, Tjia J, Lapane $\mathrm{KL}$. The role of limited English proficiency and access to health insurance and health care in the Affordable Care Act era. Health Equity. 2020;4:509-17.

6. Sentell T, Braun KL. Low health literacy, limited English proficiency, and health status in Asians, Latinos, and other racial/ethnic groups in California. J Health Commun. 2012;17:82-99.

\section{ACKNOWLEDGEMENTS}

This work was supported by the Department of Pediatrics of The Children's Hospital of Philadelphia. In addition, D.D.F. reports receiving research funding from the Agency for Healthcare Research and Quality (K08HS027468), from two contracts with the Centers for Disease Control and Prevention, and from the Children's Hospital of Philadelphia. D.M.W reports receiving research funding from the National Institute of Child Health and Human Development (K23HD102526).

\section{AUTHOR CONTRIBUTIONS}

DMW conceptualized and designed the study, drafted the manuscript, and approved of the final version of the manuscript. AMM helped acquire and analyze the data, critically revised the manuscript for intellectual content and approved of the final version. SCH, DDF, SAL, and MAE helped interpret the data, critically revised the manuscript for intellectual content and approved the final version. HHB helped design the study, acquired and analyzed the data, critically revised the manuscript for intellectual content and approved of the final version. All authors agree to be accountable for all aspects of the work.

\section{COMPETING INTERESTS}

The authors declare no competing interests.

\section{ADDITIONAL INFORMATION}

Correspondence and requests for materials should be addressed to Diana MontoyaWilliams

Reprints and permission information is available at http://www.nature.com/ reprints

Publisher's note Springer Nature remains neutral with regard to jurisdictional claims in published maps and institutional affiliations. 